\title{
Erratum
}

\section{Erratum to: Mueller-Navelet jets in next-to-leading order BFKL: theory versus experiment}

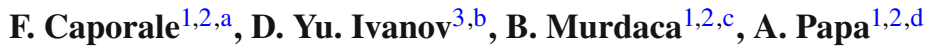 \\ ${ }^{1}$ Dipartimento di Fisica, Università della Calabria, Arcavacata di Rende, 87036 Cosenza, Italy \\ ${ }^{2}$ Istituto Nazionale di Fisica Nucleare, Gruppo collegato di Cosenza, Arcavacata di Rende, 87036 Cosenza, Italy \\ ${ }^{3}$ Sobolev Institute of Mathematics and Novosibirsk State University, 630090 Novosibirsk, Russia
}

Published online: 12 November 2015

(C) The Author(s) 2015. This article is published with open access at Springerlink.com

\section{Erratum to: Eur. Phys. J. C (2014) 74:3084 DOI 10.1140/epjc/s10052-014-3084-z}

Due to a mere transcription error, the content of Tables 7 and 8 under the columns labeled with $(a)$ does not correspond to data points in the plots of Fig. 3, which give instead the correct results of the calculation.

The corrected Tables 7 and 8 are given below. In passing, we specified that the coefficients $C_{0}, C_{1}, C_{2}$ and $C_{3}$ given in Table 7 are measured in nb. Also the coefficients $C_{0}$ and $C_{1}$ given in Table 6 are measured in nb.
Moreover, we observe that the lower limits in the integrations over $y_{1}$ and $y_{2}$ of Eq. (13) were reported as $y_{1, \min }=$ $y_{2, \min }=0$, while the correct values, as used in the numerical analyses, are instead $y_{1, \min }=y_{2, \min }=-4.7$.

Table $7 C_{0}, C_{1}, C_{2}$ and $C_{3}$ in the representation NLA 1 with the BLM method, in both variants $(a)$ and $(b)$

\begin{tabular}{|c|c|c|c|c|c|c|c|c|}
\hline \multirow[t]{2}{*}{$Y$} & \multicolumn{2}{|l|}{$C_{0}(\mathrm{nb})$} & \multicolumn{2}{|l|}{$C_{1}(\mathrm{nb})$} & \multicolumn{2}{|l|}{$C_{2}(\mathrm{nb})$} & \multicolumn{2}{|l|}{$C_{3}(\mathrm{nb})$} \\
\hline & (a) & (b) & (a) & (b) & (a) & (b) & (a) & (b) \\
\hline 3 & 2240.88 & 2267.12 & 2150.07 & 2180.83 & 1834.76 & 1860.73 & 1539.24 & 1578.51 \\
\hline 4 & 794.934 & 814.956 & 707.761 & 726.72 & 543.584 & 567.018 & 433.784 & 451.898 \\
\hline 5 & 237.577 & 252.277 & 198.863 & 206.239 & 138.340 & 146.97 & 101.337 & 109.352 \\
\hline 6 & 61.6366 & 64.3728 & 45.8401 & 47.901 & 28.7511 & 31.1006 & 19.7234 & 21.5774 \\
\hline 7 & 11.1072 & 11.7626 & 7.60735 & 7.99795 & 4.3031 & 4.73926 & 2.73730 & 3.0664 \\
\hline 8 & 0.96651 & 1.05596 & 0.63085 & 0.67637 & 0.32757 & 0.36776 & 0.19508 & 0.22453 \\
\hline 9 & 0.00911 & 0.01119 & 0.00693 & 0.00742 & 0.00334 & 0.00385 & 0.00188 & 0.00224 \\
\hline
\end{tabular}

The online version of the original article can be found under doi:10.1140/epjc/s10052-014-3084-z.

\footnotetext{
a e-mail: francesco.caporale@ fis.unical.it

be-mail: d-ivanov@math.nsc.ru

c e-mail: beatrice.murdaca@ fis.unical.it

de-mail: alessandro.papa@fis.unical.it
} 
Table $8 C_{1} / C_{0}, C_{2} / C_{0}, C_{3} / C_{0}, C_{2} / C_{1}, C_{3} / C_{2}$ in the representation NLA 1 with the BLM method, in both variants $(a)$ and $(b)$

\begin{tabular}{|c|c|c|c|c|c|c|c|c|c|c|}
\hline \multirow[t]{2}{*}{ Y } & \multicolumn{2}{|l|}{$C_{1} / C_{0}$} & \multicolumn{2}{|l|}{$C_{2} / C_{0}$} & \multicolumn{2}{|l|}{$C_{3} / C_{0}$} & \multicolumn{2}{|l|}{$C_{2} / C_{1}$} & \multicolumn{2}{|l|}{$C_{3} / C_{2}$} \\
\hline & (a) & (b) & (a) & (b) & (a) & (b) & (a) & (b) & (a) & (b) \\
\hline 3 & 0.9595 & 0.9619 & 0.8188 & 0.8207 & 0.6869 & 0.6963 & 0.8533 & 0.8532 & 0.8389 & 0.8483 \\
\hline 4 & 0.8903 & 0.8917 & 0.6838 & 0.6958 & 0.5457 & 0.5545 & 0.7680 & 0.7802 & 0.7980 & 0.7970 \\
\hline 5 & 0.8370 & 0.8175 & 0.5823 & 0.5826 & 0.4265 & 0.4335 & 0.6957 & 0.7126 & 0.7325 & 0.7440 \\
\hline 6 & 0.7437 & 0.7441 & 0.4465 & 0.4831 & 0.3200 & 0.3352 & 0.6272 & 0.6493 & 0.6860 & 0.6938 \\
\hline 7 & 0.6849 & 0.6799 & 0.3874 & 0.4029 & 0.2464 & 0.2607 & 0.5657 & 0.5926 & 0.6361 & 0.6470 \\
\hline 8 & 0.6602 & 0.6405 & 0.3389 & 0.3483 & 0.2018 & 0.2126 & 0.5134 & 0.5437 & 0.5955 & 0.6105 \\
\hline 9 & 0.7604 & 0.6634 & 0.3670 & 0.3441 & 0.2065 & 0.2005 & 0.4826 & 0.5187 & 0.5627 & 0.5826 \\
\hline
\end{tabular}

Acknowledgments We thank F.G. Celiberto for noticing the mismatch between the content of the wrong Tables 7 and 8 and the plots in Fig. 3 and for checking all entries in these tables by an independent FORTRAN program.

Open Access This article is distributed under the terms of the Creative Commons Attribution 4.0 International License (http://creativecomm ons.org/licenses/by/4.0/), which permits unrestricted use, distribution, and reproduction in any medium, provided you give appropriate credit to the original author(s) and the source, provide a link to the Creative Commons license, and indicate if changes were made.

Funded by SCOAP S $^{3}$ 